\title{
Switching of single-electron oscillations in dual-gated nanocrystalline silicon point-contact transistors
}

M. Khalafalla ${ }^{(a)}$, H. Mizuta $^{(b),(c)}$, Z. A. K. Durrani ${ }^{(a),(c)}$

(a) Microelectronics Research Centre, University of Cambridge, Madingley Road, Cambridge CB3 OHE, United Kingdom

(b) Hitachi Cambridge Laboratory, Hitachi Europe Ltd., Madingley Road, Cambridge CB3 OHE, United Kingdom

(c) CREST JST (Japan Science and Technology), Shibuya TK Bldg., 3-13-11 Shibuya, Shibuya-ku, Tokyo 150-0002, Japan

We have investigated single electron transport in dual gate point-contact transistors fabricated in $40 \mathrm{~nm}$ thick nanocrystalline silicon thin films, where the grains are 10 $\mathrm{nm}$ to $30 \mathrm{~nm}$ in size. The devices (Fig 1) consist of $30 \mathrm{~nm}$ (length) $\times 30 \mathrm{~nm}$ (width) point contacts fabricated between sources and drain regions, with an in-plane gate on each side. The grains are isolated by potential barriers formed at the grain boundaries, and show strong single electron charging effects. We observe, at $4.2 \mathrm{~K}$, switching and overlap of the Coulomb oscillation peak lines as a function of the gate voltages and at a fixed source-drain voltage as shown by broken line circles in Fig 2. These may be attributed to the switching of the potential energy in the grains at the point contact due to the charging of another, capacitively coupled, grain by single electrons.

We use single electron Monte Carlo simulation of a two-island circuit model (Fig 3) to simulate our results and explain further the switching and overlap of the oscillation peak lines. One of the islands is isolated by a tunnel junction on either side and contributes directly to the current transport. The second island is coupled capacitively to the first island and is also coupled weakly to the source. We investigate electron transport through the system as a function of circuit parameters such as the interisland coupling capacitance $\mathrm{C}_{\mathrm{f}}$, the gate capacitance, and the tunnel resistance. The results show switching and overlap of the peaks lines (white regions in Fig 4), which depend strongly on the coupling capacitance $\mathrm{C}_{\mathrm{f}}$. 


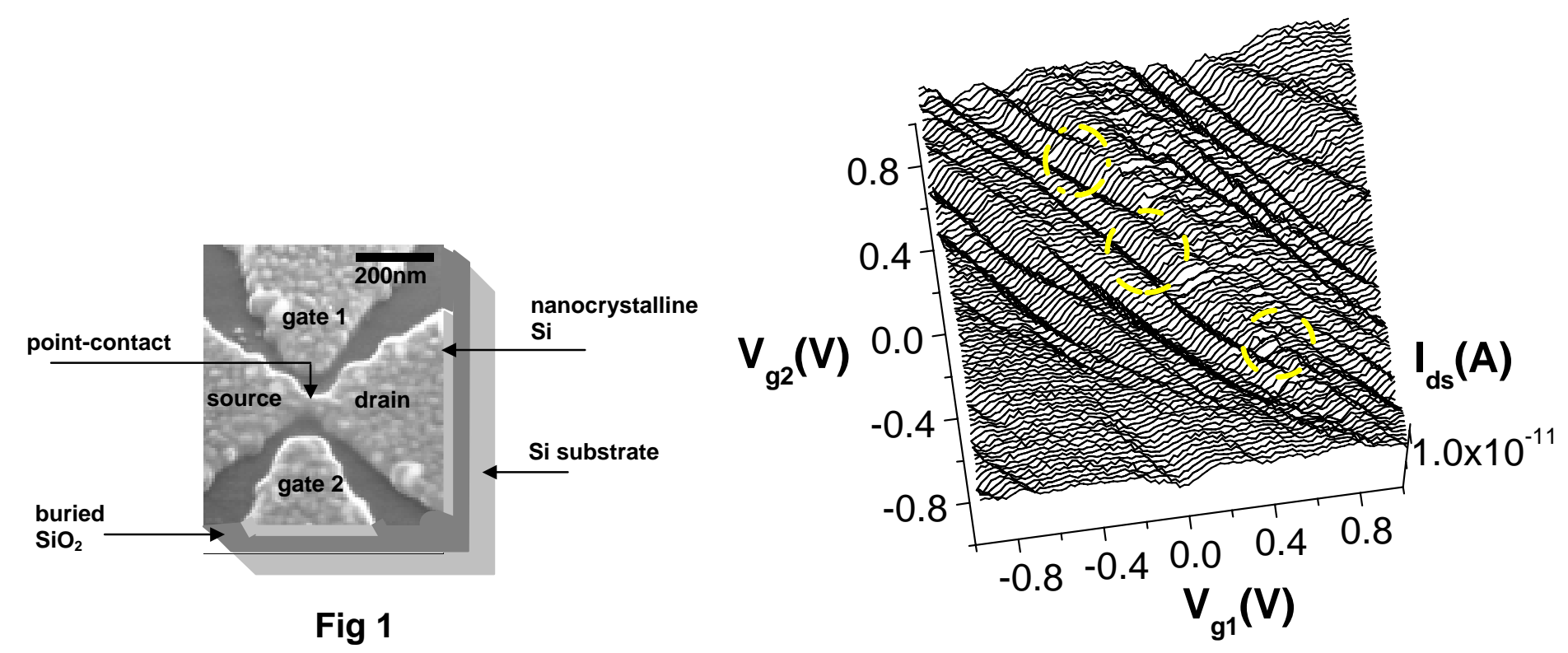

Fig 2. Coulomb oscillations as a function of the side gate voltages $\mathrm{V}_{\mathrm{g} 1}$ and $\mathrm{V}_{\mathrm{g} 2}$.

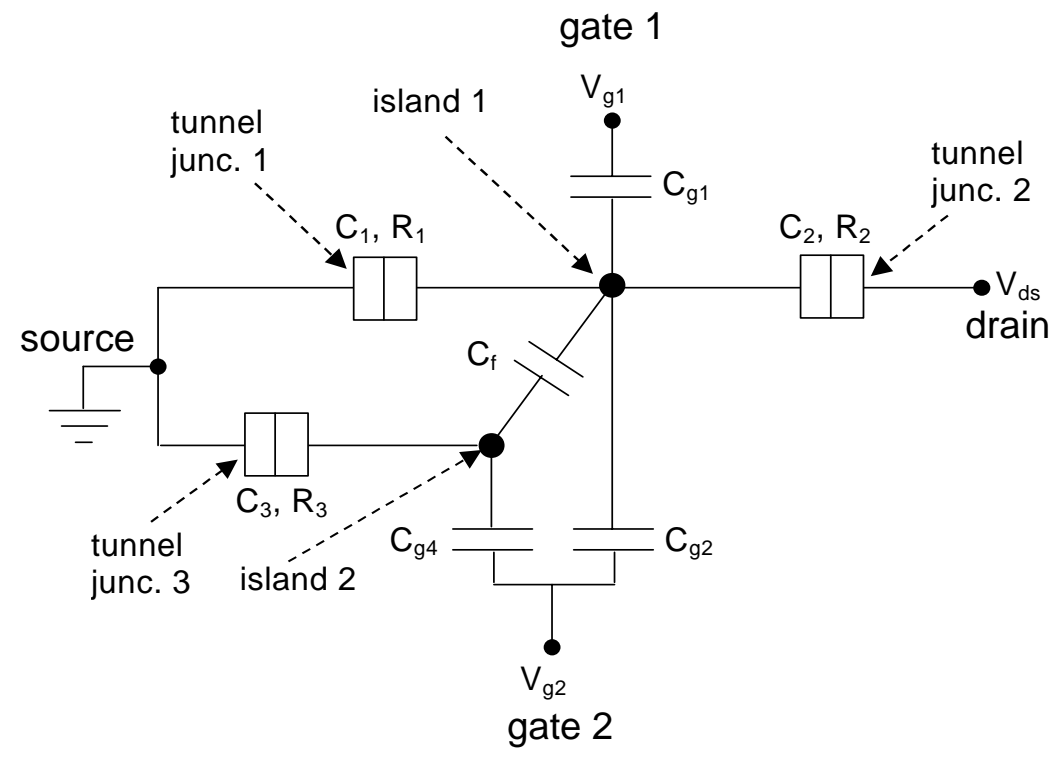

Fig 3. Simulation circuit.
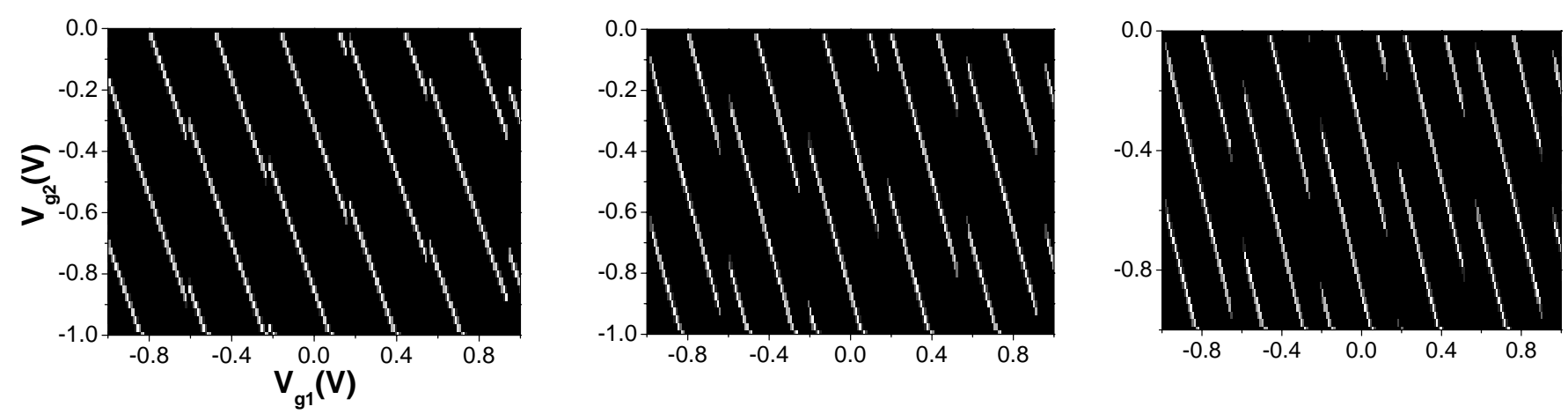

Fig 4. Simulation results. Black regions; zero current. White regions; oscillation peaks. $\mathrm{C}_{\mathrm{f}}$ is the coupling capacitor. 